\title{
On ERT and MERT-Rings
}

\section{Zubayda M. Ibraheem}

zubaida_almulla@yahoo.com

Dept. Of Mathematics,

College of Computer and Mathematical Sciences,

University of Mosul, Iraq

Received on: $26 / 06 / 2002$

Accepted on: 01/09/2002

\section{ABSTRACT}

The main purpose of this paper is to study ERT and MERT rings, in order to study the connection between such rings and IIregular rings.

Keyword: MERT-Rings, ERT-Rings and $\pi$-Regular Rings

$$
\begin{aligned}
& \text { حول الحلقات من النوع ERT و } \\
& \text { زبيدة محمد إبراهيم } \\
& \text { كلية علوم الحاسوب والرياضيات }
\end{aligned}
$$

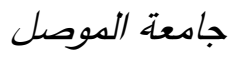

$$
\begin{aligned}
& \text { تاريخ قبول البحث: 2002/09/01 } \\
& \text { تاريخ استلام البحث: 2002/06/26 }
\end{aligned}
$$

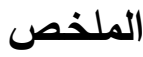

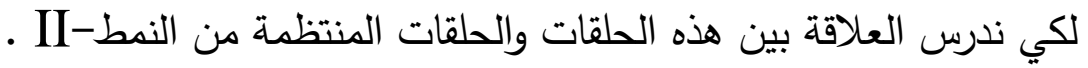

$$
\begin{aligned}
& \text { الكلمات المفتاحية: حلقات من النمط-MERT , حلقات من النم-ERT , حلقات منتظمة } \\
& \text { من النمط- }
\end{aligned}
$$




\section{1- Introduction:}

Throughout this paper, $R$ denotes an associative ring with identity, and all modules are unitary right R-module. Recall that; 1 - An ideal $\mathrm{I}$ of the ring $R$ is essential if $\mathrm{I}$ has a non-zero intersection with every non-zero ideal of $R ; 2$ - A ring $\mathrm{R}$ is said to be $\prod$-regular if for every $a$ in $R$ there exist a positive integer $n$ and $b$ in $R$ such that $\mathrm{a}^{\mathrm{n}}=a^{n} b a^{n} 3$ - A right $\mathrm{R}$-module $\mathrm{M}$ is said to be GP- injective if, for any $0 \neq a \in R$, there exists a positive integer $\mathrm{n}$ such that $\mathrm{a}^{\mathrm{n}} \neq 0$ and any right $\mathrm{R}$-homomorphism of $a^{n} R$ into $M$ extends to one of R into M. 4- For any element $a$ in $R, r(a), I(a)$ denote the right annihilator of $a$ and the left annihilator of a, respectively.

\section{2- ERT-R1NGS:}

Following [3J, a ring $R$ is said to be ERT-ring if every essential right ideal of $R$ is a two-sided ideal.

\section{Definition 2-1:}

A ring $R$ is said to be right weakly regular if for all $a$ in $R$, there exists $b$ in $R a R$ such that $a=a b$, or equivalently every right ideal of $\mathrm{R}$ is idempotent.

We begin this section with the following main result:

\section{Theorem 2.2:}

If $R$ is ERT-ring with every essential right ideal is idempotent, then $R$ is weakly regular.

\section{Proof:}

For any $a \in R$, if $R a R$ not essential, then there exists an ideal I, such that $\quad K=R a R \oplus \mathrm{I}$ is essential then $K=K^{2}$.

In order to prove that $R$ is weakly regular, we need to prove $\operatorname{RaR}=(\operatorname{RaR})^{2}$.

For a $\in \mathrm{K}$, we have $\mathrm{a} \in \mathrm{K}^{2}$, that is $a \in(\mathrm{RaR} \oplus \mathrm{I})^{2}$

Thus $a=\left(r a r^{\prime}+i\right)\left(s a s^{\prime}+i^{\prime}\right)$ for some $r, \mathrm{r}^{\prime} s, s^{\prime} \in R$ and i, $i^{\prime} \in \mathrm{I}$. 
This implies that $a=\left(\right.$ rar $\left.^{\prime}+i\right) \operatorname{sas}^{\prime}+\left(\right.$ rar $\left.^{\prime}+i\right) i^{\prime}$

$$
=\operatorname{rar}^{\prime} s a s^{\prime}+i s a s^{\prime}+\left(\operatorname{rar}^{l}+i\right) i^{\prime}
$$

but $i s a s^{\prime} \in \mathrm{I} \cap \mathrm{RaR}=0$, also we have (rar' $\left.+i\right) i^{\prime} \in \operatorname{RaR} \cap \mathrm{I}=0$.

Therefore $a=\left(\right.$ rar $\left.^{\prime}\right)\left(\right.$ sas $\left.^{\prime}\right) \in(R a R)^{2}$, this implies that $R a R$ $\subseteq(R a R)^{2}$ Thus $R a R=(R a R)^{2}$, this proves that $R$ is weakly regular ring.

Following [2], the singular submodule of $R$ is

$Y(R)=\{y \in R, r(y)$ is essential right ideal of $R$.

\section{Theorem 2.3:}

Let $R$ be a semi-prime ERT right GP-injective ring. Then $R$ is a right non singular.

\section{Proof:}

Let $E$ be an essential right ideal of $R$. Then $\mathrm{E}$ is a two-sided ideal, and hence $l(E)$ is a two-sided ideal $o f R$.

Now $(l(E) \cap E)^{2} \subseteq(E) E=0$.

Since $R$ is semi-prime, then $l(E) \cap E=0$, whence $l(E)=0$. This proves that $R$ is right non singular.

\section{3- MERT-R1NGS:}

Following [3], a ring $R$ is said to be MERT-ring if every maximal essential right ideal of/? is a two-sided ideal.

\section{Theorem 3.1:}

Let $R$ be an MERT-ring, if for any maximal right ideal $\mathrm{A} / \mathrm{of} \mathrm{R}$, and for any $b$ e $M, b R / b M$ is GP-injective, then $R$ is strongly Pi-regular ring.

\section{Proof:}

Let $b$ be a non-zero element in R, we claim that $b^{n} r+r\left(b^{\prime \prime}\right)=R$. If $b^{n} r+r\left(b^{n}\right) \neq R$, let $\mathrm{M}$ be a maximal right ideal containing $b^{n} r+$ $r\left(b^{n}\right)$. Then $M$ is essential right ideal of $\mathrm{R}$. 
If $b R=b M$, then $\mathrm{b}=\mathrm{bc}$, for some $c$ in $\mathrm{M}$, this implies $(1-c) \in \mathrm{r}(\mathrm{b}) \subset r\left(b^{n}\right) \subset M$, therefore $1 \in M$, this contradics $\mathrm{M} \neq R$.

Now, since $R / M \cong b R / b M$. Then $\mathrm{R} / M$ is GP-injective.

Now, define $f: b^{n} R \rightarrow R / M$ by $f\left(b^{n} r\right)=r+\mathrm{M}$, note that $\mathrm{f}$ is a well-defined R-homomorphism.

Since R/M is GP-injective, then there exists $c \in R$, such that:

$1+\mathrm{M}=\mathrm{f}\left(\mathrm{b}^{\prime \prime}\right)=c b^{\prime \prime}+\mathrm{M}$ and so $\left(1-\mathrm{c} \mathrm{b}^{\mathrm{n}}\right) \in \mathrm{M}$, since $\mathrm{b}^{\mathrm{n}} \in \mathrm{M}$, and $\mathrm{R}$ is MERT-ring, this implies that $\mathrm{M}$ is a two-sided ideal, and hence $\in \mathrm{c} b^{\prime \prime} \in \mathrm{M}$.

Thus $\mathrm{I} \in \mathrm{M}$, a contradiction.

Therefore $b^{\mathrm{n}} \mathrm{R}+\mathrm{r}\left(\mathrm{b}^{\mathrm{n}}\right)=\mathrm{R}$.

In particular $l=b^{n} u+v ; v \in r\left(b^{n}\right), u \in R$.

Thus $\mathrm{b}^{\mathrm{n}}=\mathrm{b}^{2 \mathrm{n}} u$ and therefore $\mathrm{R}$ is strongly $\prod$-regular ring.

\section{Theorem 3.2:}

If $R$ is MERT-ring with every simple singular right ideal is GP-injective, then $Y(R)=0$.

\section{Proof:}

If $Y(R) \neq 0$, by Lemma (7) of [6], there exists $0 \neq y \in Y(R)$ with $\mathrm{y}^{2}=0$. Let $L$ be a maximal right ideal of $R$, set $\mathrm{L}=y \mathrm{R}+\mathrm{r}(\mathrm{y})$, we claim that $L$ is essential right ideal of $\mathrm{R}$. Suppose this is not true, then there exists a non-zero ideal $T$ of $\mathrm{R}$ such that $\mathrm{L} \cap \mathrm{T}=(0)$. Then $\mathrm{yRT} \subseteq L T \subseteq L \cap T=0$ implies $\mathrm{T} \subseteq \mathrm{r}(\mathrm{y}) \subseteq \mathrm{L}$, so

$L \cap T=(0)$. This contradiction proves that $L$ is an essential right ideal, that is $R / L$ is

simple singular and hence $R / L$ is GP-injective.

Now; Let $f ; y R \longrightarrow R / L$ be defined by $f(y r)=r+L$, then $\mathrm{f}$ is a well-defined $R$ -

homomorphism.

Since $R / L$ is GP-injective, so $\exists c \in R$, such that $l+L=f(y)=c y+L$. Hence $l+L=c y+L$, implies that $l-c y \in L$. 
Since $R$ is MERT, then eye $L$ and thus $l \in L$, a contradiction. Therefore $Y(R)=[0]$.

Following [1], a ring $R$ is zero insertive ( briefly ZI) if for $a, b \in R, a b=0$ implies $a R b=0$.

\section{Theorem 3.3:}

Let $R$ be a ZT ring. If every simple singular rightsmodules is GP-injective which is left self-injective, then $R$ is strongly $\mathrm{H}$-regular ring.

\section{Proof:}

Since $R$ is simple singular GP-injective, then $R$ is semiprime, by Lemma (4)

of [5].

Thus for any left ideal I, $L(I) \cap l=0$.

Since $R$ is simple singular GP-injective and ZI, then $R$ is reduced and hence $r(a)=l(a)$ for any element $a$ in $R$.

Thus $l(r(a)) \cap l(a)=l(l(a)) \cap l(a)=0$.

Since $R$ is left self-injective ring, then $a R$ is a right annihilator, by Proposition (4)of[4].

Since $r(a) \subseteq r\left(a^{n}\right)$, then $a^{n} R=r\left(a^{f l}\right)$.

Now, since $R=r(l(r(a)))+r(l(a))_{9}$ then we have $R=r\left(l\left(r\left(a^{n}\right)\right)+\right.$ $r\left(l\left(\left(a^{n}\right)\right)=r\left(a^{n}\right)+a^{n} R\right.$

In particular, for some $b$ in $R$, and $\mathrm{d}$ in $r\left(a^{n}\right)$.

Thus $a^{\prime \prime}=a^{n 2} b$.

Therefore $R$ is strongly П-regular. 


\section{REFERENCES}

[1] Kirn. N.M., Nam. S.B. and Kirn. J.Y. (1999); "On simple singular GP-Injective Modules"; Communication In Algebra, 27(5), 2087-2096.

[2] Kim. N.M., Nam. S.B. and Kirn. J.Y. (1995); "On simple GP-injective Modules"; Communications In Algebra, 23(14), 5437-5444.

[3] Ming R.Y.C. (1980); "On V-Rings and Prime Rings"; Journal of Algebra. 62, 13-20.

[4] Ming R.Y.C. (1980); "On Von-Neumann regular ring"; Math. J.Okayama Univ. vol. 22. No. 2.

[5] Ming R.Y.C. (1976); "On annihilator ideals"; Math. J. Okayama Univ. 19,51-53.

[6] Ming R.Y.C. (1983); "On quasi-injectlvity and VonNeumann regularity", Montash, Math, 95, 25-32. 